\title{
Measuring earthquakes from optical satellite images
}

\author{
Nadège Van Puymbroeck, Rémi Michel, Renaud Binet, Jean-Philippe Avouac, and \\ Jean Taboury
}

\begin{abstract}
Système pour l'Observation de la Terre images are used to map ground displacements induced by earthquakes. Deformations (offsets) induced by stereoscopic effect and roll, pitch, and yaw of satellite and detector artifacts are estimated and compensated. Images are then resampled in a cartographic projection with a low-bias interpolator. A subpixel correlator in the Fourier domain provides twodimensional offset maps with independent measurements approximately every $160 \mathrm{~m}$. Biases on offsets are compensated from calibration. High-frequency noise $\left(0.125 \mathrm{~m}^{-1}\right)$ is $\sim 0.01$ pixels. Low-frequency noise (lower than $0.001 \mathrm{~m}^{-1}$ ) exceeds 0.2 pixels and is partially compensated from modeling. Applied to the Landers earthquake, measurements show the fault with an accuracy of a few tens of meters and yields displacement on the fault with an accuracy of better than $20 \mathrm{~cm}$. Comparison with a model derived from geodetic data shows that offsets bring new insights into the faulting process. () 2000 Optical Society of America

OCIS codes: $\quad 100.0100,100.2000,100.6640,100.2960,280.0280$.
\end{abstract}

\section{Introduction}

Earthquakes produce static displacements of the ground that can be observed near the fault that slipped during a seismic rupture. Measurement of the displacements is a key issue in seismotectonics, because it brings insight about the geometry of the ruptured fault and the energy released by the earthquake. Such measurements are generally performed with geodetic techniques that can provide only a sparse covering, because the areas of coseismic ground displacement are not known a priori. Satellite imagery is naturally suited for such measurements, because it regularly provides comprehensive and detailed images of the ground with high radiometric and geometric quality. The displacement field can be measured by comparison of images acquired before and after the event. Synthetic aperture radar (SAR) images were actually shown to be useful for this application with either interferometry or offsets. ${ }^{1,2}$ SAR interferometry exploits the phase

The authors are with the Laboratoire de Détection et de Géophysique, Télédéction et Risque Sismique, Département Analyse et Surveillance de l'Environnement, Commissariat à l'Energie Atomique/DAM, BP 12, 91680 Bruyères le Châtel, France. R. Michel's e-mail address is michel@ldg.bruyeres.cea.fr.

Received 14 December 1999; revised manuscript received 3 May 2000.

0003-6935/00/203486-09\$15.00/0

(C) 2000 Optical Society of America difference of complex images and yields the nearvertical component of the displacement with an accuracy of $\sim 1 \mathrm{~cm}$. This technique has severe limitations that are mainly due to data decorrelation and signal saturation, and it does not generally provide measurements in the near-fault area where large displacements occur. The technique of offsets provides a measurement of the ground displacement from the analysis of the geometrical deformation between the two amplitude images. Offset were first measured with SIR-C ( $L$-band; SIR is Shuttle Imaging Radar) and European Remote-Sensing Satellite (ERS) radar amplitude images.2,3 Measurements must be performed with subpixel accuracy, because the amplitude of the ground displacement (a few meters for large earthquakes) is typically lower than the resolution of the images. SAR offsets provide two components of the displacement field with an accuracy of a few tens of centimeters and independent measurement approximately every $160 \mathrm{~m}$. This technique is mainly limited by the pixel size ( $\sim 10 \mathrm{~m}$ for SIR-C and ERS images). In this paper we present a method to measure the displacement of the ground induced by an earthquake from optical satellite imagery and apply it to the Landers earthquake (7.3 Mw, June 1992, California) with Système pour l'Observation de la Terre (SPOT) panchromatic images. Such an approach was first explored by Crippen, ${ }^{4}$ and it may be of great interest, because thousands of images of the ground are available and because metric resolution images will soon be deliv- 
ered by commercial satellites. We first present the characteristics of the SPOT push-broom imagery that are of interest in this study. The origin of offsets between two images is analyzed, and the images are then resampled with a digital elevation model (DEM) so that only the offsets due to the earthquake remain. The influence of the interpolator used to resample the images is analyzed. A subpixel correlator adapted to the characteristics of both the images and the ground displacement is then presented. The limits and potential of the technique are then evaluated on the basis of the Landers earthquake case study by comparison of offsets with a detailed in situ cartography of the fault ${ }^{5}$ and an elastic modeling of the ground deformation constrained from numerous geodetic data. ${ }^{6}$

\section{Système pour l'Observation de la Terre Panchromatic Push-Broom Imagery}

In this section we describe the characteristics of SPOT panchromatic imagery that are useful for the study. The basic principles are similar when other push-broom devices such as the Indian Remote Sensing satellite are used. Two high-resolution visible (HRV) push-broom optical devices onboard the SPOT platforms (SPOT satellites 1, 2, 3, and 4) provide panchromatic images of a $60-\mathrm{km}$-wide ground area with a resolution of $\sim 10 \mathrm{~m}$ (Fig. 1). ${ }^{7}$ The optical device includes a Schmidt-like telescope $(f=1.082$ $\mathrm{m}, F / 3.3$ ); a tunable mirror that allows for selection of the location of the scene center; and the DIVOLI (Diviseur Optique de Ligne), which connects four 1500-pixel CCD arrays to provide a 6000-pixel line. Lines are acquired successively during the motion of the satellite. The optical center is at the center of gravity of the platform that is submitted to roll, pitch, and yaw. SPOT images are composed of $6000 \times$ 6000 pixels, and digitization is 1 byte (though the effective dynamic is often lower than 255 levels). Pixel gains are compensated to get raw (1A) images. ${ }^{7}$ Parameters of acquisition are known, including attitude of the platform, and lead to absolute location of the scene with an accuracy of $\sim 1 \mathrm{~km}$. The position in the image of a given point $M$ on the ground can be analytically derived from the parameters of the images and the elevation of $M .^{7}$ It is noteworthy that SPOT images experience aliasing, because the modulation transfer function of the optical device is not zero (equal to $\sim 0.2$ ) at the sampling frequency of the CCD detector. ${ }^{7}$

\section{Origin and Compensation of Offsets}

\section{A. Origin of Offsets}

Two images of the same area acquired during two different orbits present offsets that depend on (Fig. 2).

- Distance between the orbits.

- Viewing angles.

- Topography (stereoscopic effect).

- Attitude of the platform: roll, yaw, pitch.
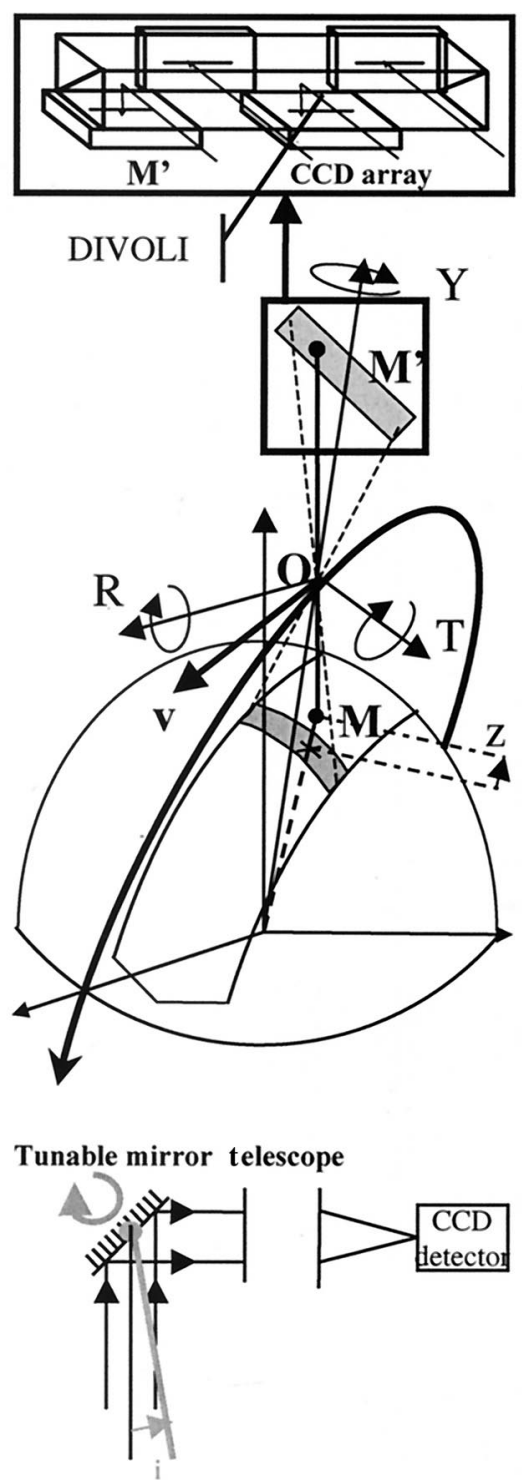

Fig. 1. SPOT push-broom imagery: Lines are acquired successively as SPOT satellite moves along its orbit. Each line of 6000 pixels is acquired with $4 \times 1500$ CCD detectors. Resolution is $\sim 10 \mathrm{~m}$. A mirror allows for tuning of viewing angle $i$. The optical center is at the center of gravity of SPOT that is submitted to roll, pitch, and yaw (R, $\mathbf{P}$, and $\mathbf{Y}$ rotation vectors).

- Geometry of the detectors.

- Displacement of the ground (due to the earthquake).

A set of images acquired at different dates also include temporal decorrelation induced by ground changes, seasonal effects, and difference in solar angles. The source of decorrelation leads to noise in the measurement of offsets. Measuring the topography from a stereoscopic pair of SPOT images has been investigated intensively. ${ }^{8}$ The stereoscopic effect leads to offsets characterized by strong gradients particularly in areas with contrasting topographics. Those offsets are thus difficult to measure, and stan- 


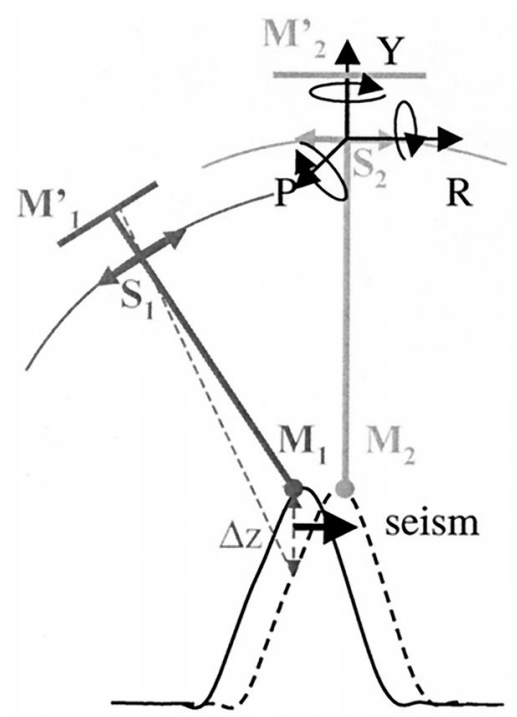

Fig. 2. Offset measured from correlation of images 1 and $2 ; M^{\prime}{ }_{1}$ $M^{\prime}{ }_{2}$ depends mainly on orbits, parameters of acquisition, topography, attitude $(R, P, Y)$, detectors, and ground displacement $M_{1} M_{2}$. Error $\Delta z$ in the DEM yields biased estimates of $M^{\prime}{ }_{1}$ and $M^{\prime}{ }_{2}$.

dard methods yield measurements with an accuracy of $\sim 0.5$ pixels (rms).

\section{B. Compensation of Offsets}

To measure ground displacement, we have to model and compensate the other sources of offsets. Positions $M_{1}$ and $M_{2}$ in the images of a point $M$ on the ground (Fig. 2) can be modeled analytically from the equation of the image. ${ }^{7}$ We use the parameters of the images and a DEM to model and compensate the stereoscopic effect (Subsection 3.B.1). Geometrical effects that are due to detector artifacts are estimated and compensated (Subsection 3.B.2). To minimize the stereoscopic effect, we use images with nearvertical incidence so that differences in viewing angle $\Delta i$ do not exceed $3^{\circ}$ (Table 1 ).

\section{Influence of Digital Elevation Model Quality}

An error $\Delta z$ on the estimate of the elevation of a point $M$ on the ground will lead to errors $\Delta M$ in the estimate of corresponding locations in the image that can be estimated as

$$
\Delta M \approx \Delta z f i /\left(h p_{x}\right) \quad \text { (pixels) }
$$

Table 1. SPOT Images Used to Study the Landers Earthquake

\begin{tabular}{lccc}
\hline \multicolumn{1}{c}{ Images } & $\begin{array}{c}\text { SPOT2 } \\
\text { Panchromatic }\end{array}$ & $\begin{array}{c}\text { SPOT2 } \\
\text { Panchromatic }\end{array}$ & $\begin{array}{c}\text { SPOT2 } \\
\text { Panchromatic }\end{array}$ \\
\hline Date & 27 July 1991 & 25 July 1992 & 10 October 1993 \\
Pixel size & $10 \mathrm{~m}$ & $10 \mathrm{~m}$ & $10 \mathrm{~m}$ \\
KJ $^{a}$ & & $544-281$ & \\
Viewing angle & $-1.6^{\circ}$ & $-2.6^{\circ}$ & $2.6^{\circ}$ \\
Optical device & HRV2 & HRV1 & HRV1 \\
\hline
\end{tabular}

${ }^{a} \mathrm{KJ}$, location of the scene.

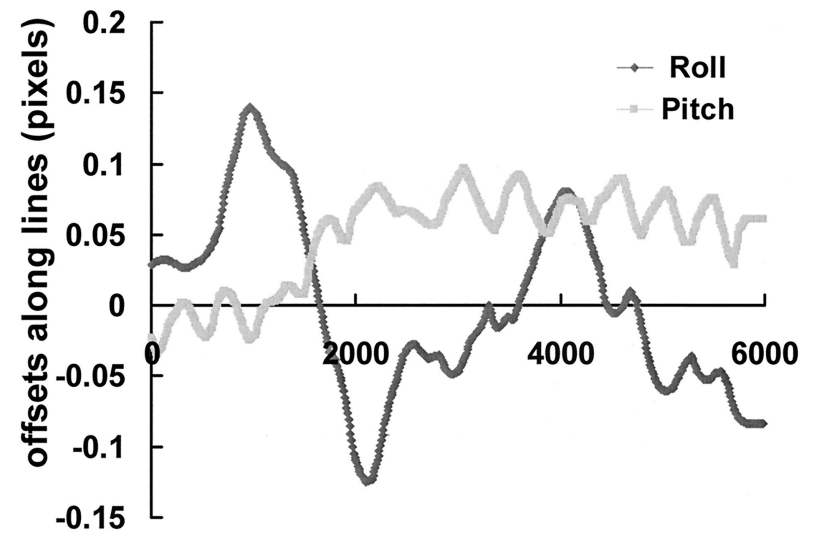

position (lines)

Fig. 3. Example of along line offsets induced by the attitude of SPOT. Modeling from onboard measurements of roll, pitch, and yaw allows for partial compensation of that effect. Residual error due to aliased measurements of roll, pitch, and yaw results in low-frequency noise in offset fields.

where $f$ is the focal length, $h$ the altitude of the platform $(\sim 800 \mathrm{~km}), i$ the viewing angles, and $p_{x}$ the pixel size $(13 \mu \mathrm{m})$ (Fig. 2). $\Delta z=20 \mathrm{~m}$ (the typical value for a DEM derived from SPOT images), and $i=3^{\circ}$ leads to $\Delta M=0.1$ pixels. The influence of that significant source of noise will be reduced by means of averaging in the correlation procedure (see Subsection 5.A).

\section{Effect of Roll, Pitch, and Yaw}

The attitude of the platform leads to geometrical deformations of the images. Independent measurements of the velocity of the roll, pitch, and yaw are given approximately every 80 lines. Initial values of roll, pitch, and yaw are unknown. We first estimate them by using a least-squares procedure from tie points between the DEM and the raw (1A) images: This procedure minimizes the distance between the positions estimated from the equation of the image and the tie points. It provides the initial parameters with an accuracy of $\sim 0.5 \times 10^{-5}$ rad with $\sim 20$ tie points. Geometrical deformations $\Delta l$ and $\Delta c$ along lines and columns $(l, c)$ induced by roll, pitch, and yaw $(R, P, Y)$ can be modeled as

$$
\begin{aligned}
\Delta l & =P h / p_{y}+c \sin (Y), \\
\Delta c & =f R / p_{x}+c[1-\cos (Y)] \quad \text { (pixels). }
\end{aligned}
$$

The effect of the pitch and yaw on offsets may exceed few tenths of a pixel (Fig. 3). Corresponding offsets show instantaneous frequencies that fairly correspond to those described in Ref. 7. Unfortunately, provided measurements of roll, pitch, and yaw are undersampled so that the compensation modeled from Eq. (2) may be biased. However, resulting errors will not lead to discontinuities in offset fields and thus should not prevent measurements of near-fault displacements induced by earthquakes. 
(a)

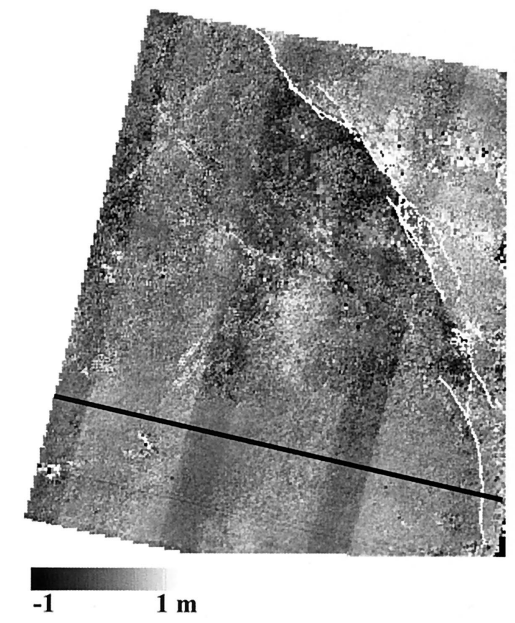

(b)

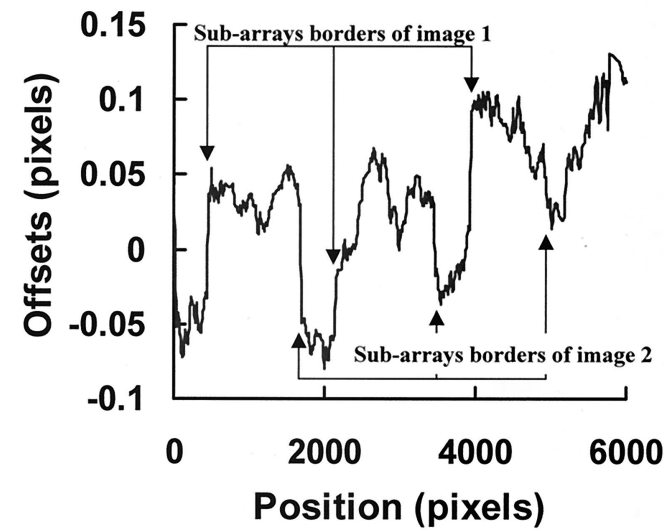

Fig. 4. (a) Component along columns of offset fields derived from images 1 and 2 (Table 1). Linear discontinuities are induced by misalignment of the four CCD arrays composing the detection lines. White curves, cartography of surface break by Sieh et al. ${ }^{5}$ Black line, location of profile in (b).

\section{Compensation of Detector Artifacts}

As noted in Section 2, detectors are composed of four subarrays of 1500 pixels each. The pixels located at the borders of two consecutive subarrays partially overlap to avoid gaps in the image of the scene. Those geometrical artifacts yield discontinuities in the offset fields located at the position corresponding to the subarray borders [Fig. 4(a)]. The discontinuities corresponding to each image are not located at the same positions, because of the difference in the scene centers. We measure those discontinuities on profiles in the offsets [Fig. 4(b), Table 2]. The discontinuities depend on the detector. Their ampli-

Table 2. Amplitude of Misalignments of SPOT 2 HVR1 and HVR2 Subarray Detectors ${ }^{a}$

\begin{tabular}{cccc}
\hline Position (pixels) & 1500 & 3000 & 4500 \\
\hline Discontinuity (pixels) & 0.075 & 0.045 & -0.05 \\
$\quad$ HRV1 & & & -0.065 \\
$\begin{array}{l}\text { Discontinuity (pixels) } \\
\text { HRV2 }\end{array}$ & -0.10 & -0.05 & \\
\hline
\end{tabular}

${ }^{a}$ Values are computed from the profile of Fig. 4(b). Misalignments yield discontinuities in offsets located every 1500 pixels. tudes do not exceed 0.2 pixels, and they can be measured with an accuracy of better than 0.01 pixels. Once determined for a given detector we account for the values of those discontinuities in the analytical expression given position $M^{\prime}$ in the image of a point $M$ on the ground (Table 2).

\section{Interpolation of Système pour l'Observation de la Terre Images}

Images are now resampled to be in a cartographic projection. As noted in Section 2, SPOT images are undersampled, and they include aliasing. Exact interpolation to compute the radiometry at a given point on the ground from image pixels is thus not possible. We tested the bicubic spline interpolator and the sinc interpolator defined by

$$
b_{\text {interp }}(x, y)=\sum_{(c, l)} b(c, l) \frac{\sin \pi(c-x)}{\pi(c-x)} \frac{\sin \pi(l-y)}{\pi(l-y)},
$$

where $b_{\text {interp }}$ and $b$ are the interpolated and the raw images, respectively, and $(x, y)$ are the coordinates in the interpolated images. In practice the sum in Eq. (3) is restricted to a $11 \times 11$ pixel vicinity of $(x, y)$. The residual contribution of other pixels is lower than $0.1 \%$ of the digitization step and thus can be neglected.

We use the following procedure: (i) A raw SPOT image is shifted in lines and columns by subpixel values ranging from -1 to +1 pixels, with the two interpolators; (ii) at each step we measure the twodimensional offset field, using sliding $32 \times 32$ windows and the correlator described in Section 4; (iii) for each theoretical value of the offset we compute the averaged measured offset over the offset field to get the chart shown in Fig. 5. We note that the two interpolators lead to biased estimates of offsets. The bias induced by the bicubic spline interpolator is the largest and reaches 0.2 pixels. The bias depends on theoretical values of offsets, on local properties of the images, and possibly on the correlator. It does not exceed 0.05 pixels for the sinc interpolator. Below, we use that interpolator. Calibration of measurements is discussed in Subsection 5.B.

\section{Subpixel Correlation of Système pour l'Observation de la Terre Images}

\section{A. Method}

In this section we assume that the images were previously resampled according to Section 3 so that they are in a cartographic (Subsection 3.A) projection. Residual offsets remain that relate to (i) residual uncertainty in estimates of satellite parameters, (ii) errors in DEM, and (iii) deformation induced by earthquakes. Those offsets are characterized by gradients typically lower than $0.1 \%$ except within a few tens of meters of faults. They are generally smaller than the pixel size (ranging from a few tens of centimeters to a few meters). Correlation methods using sliding windows have been proved to be efficient to measure such offsets. ${ }^{2,3}$ Offsets may be de- 


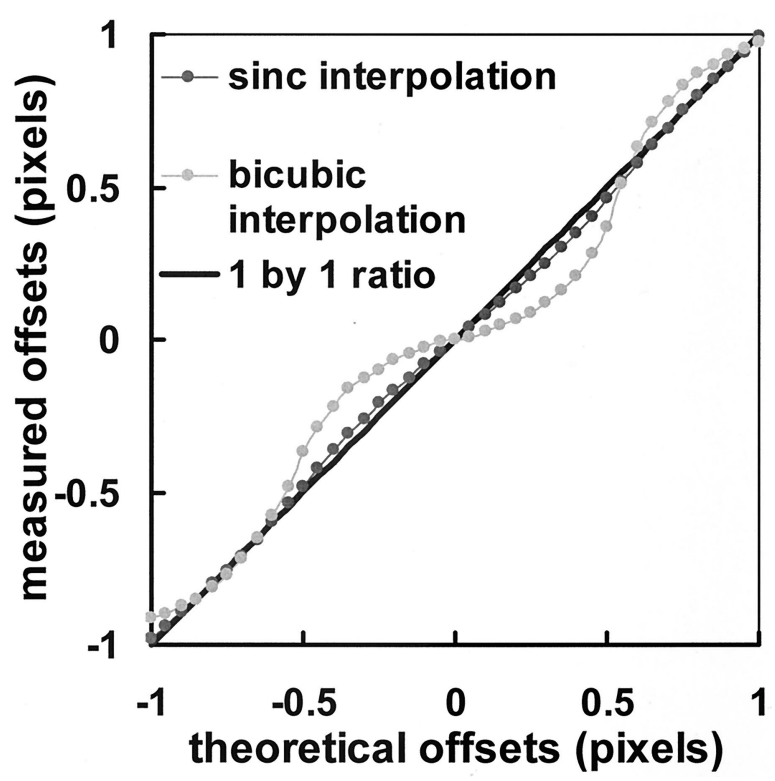

Fig. 5. Images are resampled to be in a cartographic projection to compensate all offsets except those induced by the earthquake. No exact interpolator exists to do so, because images include aliasing. The bicubic spline interpolator leads to greater biased estimate of offset than the sinc interpolator (see text for details). Residual bias is $\sim 0.02$ pixels and is postcompensated from calibration.

termined from a broad range of techniques.9,10 Recent improvements have been made to measure offsets in the Fourier domain. ${ }^{2,11}$ Those techniques analyze the phase difference of the local instantaneous frequencies of the images. In the following $A$ and $B$ denote two sliding $N \times M$ windows extracted from the images acquired, respectively, before and after the earthquake [Fig. 6(a)]. Periodograms [i.e., the amplitude of the numerical Fourier transform computed from the fast Fourier transform (FFT)] of $A$ and $B$ correspond to instantaneous frequencies of the imaged area of the ground and also to artifacts [Fig. 6(b)]. Since images are not $N \times M$ periodic, this process leads to artifacts located along the two axes corresponding to zero frequencies [vertical cross in Fig. 6(b)]. Residual errors in equalization of detectors ${ }^{7}$ result in linear artifacts with an azimuth of $\sim 12^{\circ}$ in Fig. 6(b). Undersampling of images (see Section 2) leads mainly to high-frequency artifacts. The phase difference of the numerical Fourier transform of $A$ and $B$ shows a phase ramp $\Theta$ that verifies [Fig. 6(c)]

$$
\exp j \Theta \approx \exp (2 \pi j \boldsymbol{v} \mathbf{t})
$$

where $\boldsymbol{v}$ and $\mathbf{t}$ are, respectively, the vectors corresponding to the instantaneous frequency and the translation between $A$ and $B$ that is to be determined. In addition to the phase ramp Fig. 6(c) shows artifacts that are correlated to artifacts measured in the periodograms of Fig. 6(b). This shows that the phase noise in Fig. 6(c) is not a monotonic function of periodograms: High value of the periodogram may be associated with noisy value of the phase difference.

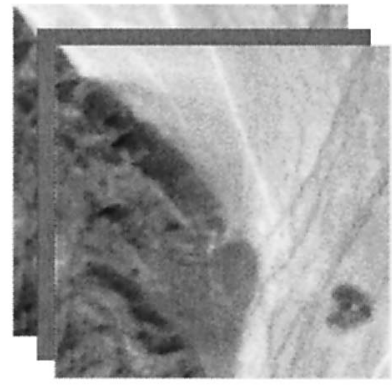

(a)

(c)

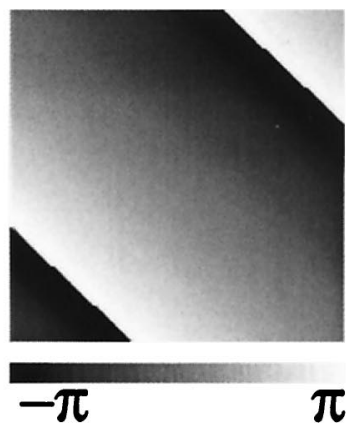

(e)

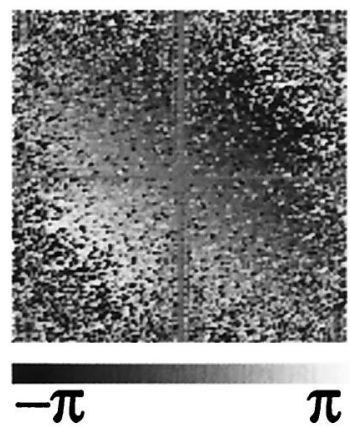

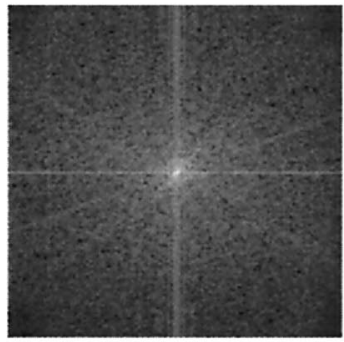

(b)

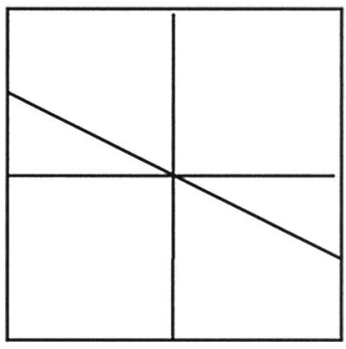

(d)
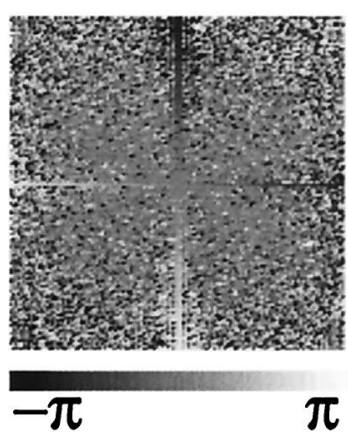

(f)
Fig. 6. Subpixel estimate of offset from phase shift. (a) Sliding windows $(N=128)$ extracted from orthorectified interpolation of SPOT images 1 and 2 (Table 1). (b) Periodogram (log scale) computed from FFT. Vertical cross is an artifact of FFT that results from non- $N \times N$ periodicity of images. $12^{\circ}$ orientated line reflects residual error in the equalization of detectors (see text). (c) Phase difference (modulo $2 \pi$ ) of FFT's. Subpixel offsets results in phase ramp. Artifacts in (b) result in artifacts in phase difference. Phase noise is not a monotonic function of periodogram. (d) $\mathrm{Bi}$ nary mask derived from (b). Measurements along the black lines are not used to estimate offsets. (e) Synthetic phase shift that best fits image (c) according to $L_{1}$ norm (see text). (f) Residual phase (c)-(e). Offset is estimated from (e). Presented offset is equal $(0.7,0.8)$ pixels; incertitude is 0.01 pixels.

That source of noise is generally not accounted for in the correlators used with SPOT images such as the classical normalized correlation function in the image domain. ${ }^{8}$ We estimate $t$ from the following procedure:

(a) We defined a mask $m$ that is zero along the direction corresponding to the linear artifacts de- 
noted in the periodogram and equal to 1 otherwise [Fig. 6(d)].

(b) We create a set of complex two-dimensional 2D phase ramps $\mathbf{i}_{t, p}$ that describe possible values of $t$ with a predefined value $p$ of the step [Fig. 6(e)].

(c) We estimate $t$ as the value that maximizes $\epsilon$ defined as

$$
\epsilon=\frac{\left|\sum m i_{t, p}^{*} \exp j \Theta\right|}{\sum m},
$$

where the sum is computed over the instantaneous frequencies.

To save time we use a two-step dichotomy procedure. The value of $p$ is first chosen as 0.1 pixels, and the phase ramps describe the values of $t$ ranging from -1 to +1 pixels. The measurement $\exp j \Theta$ is then compensated by the value of $\mathbf{i}_{t, p}$ that maximizes $\epsilon$. The value of $p$ is then reduced to 0.01 pixels, and the phase ramps describe the values of $t$ ranging from -0.1 to +0.1 . An estimate of error on the measurement can be derived from $\epsilon$. If we assume a uniform distribution of phase noise in $\Theta$, the average error $\Delta$ on the components of the measured offset can be estimated as

$$
\epsilon=\sin \left(\frac{2 \pi \Delta^{2}}{N M}\right) /\left(\frac{2 \pi \Delta^{2}}{N M}\right)
$$

Here $\epsilon$ provides insights about the level of decorrelation of $A$ and $B$ but is not a reliable estimate of errors in the measurement of ground displacement, because it does not account for sources of correlated noise such as those induced by roll, pitch, and yaw.

\section{B. Calibration}

Bias induced by the interpolator and the correlator also depend on the local characteristics of the images: Areas with dense spectra lead to low bias, whereas homogeneous areas lead to large bias (greater than 0.1 pixels). Moreover, biases are not the same along the lines and the columns. Postcompensation of bias thus required local analysis. We estimate biases from the procedure presented in Subsection 3.C: For each sliding window we plot the measured offset as a function of the theoretical values to get a chart similar to the averaged chart of Fig. 5. This chart is then used to estimate and compensate the bias on the measurements.

Compensation of the effects of roll, pitch, and yaw also induces bias, because measurements of those quantities are undersampled (Subsection 3.B.2). This bias leads to low-frequency artifacts on the offsets fields [Fig. 4(a)]. We assume that the far-field displacement induced by the earthquake is negligible, and we remove from offset fields a first-order polynomial function determined by a least-squares procedure. Higher-order terms limit the lowfrequency accuracy of the measurements.

We assessed the quality of our measurements by computing the offsets, using two images both acquired after the earthquake (Table 1). Theoretical

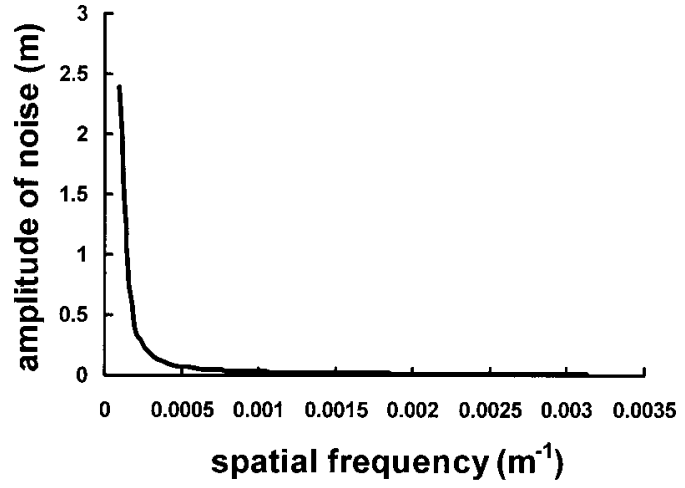

Fig. 7. Chart derived from offset fields computed from SPOT images of the Landers area including no ground displacement (see text). Noise on offset fields varies with spatial frequency mainly because of low-frequency errors in modeling influence of roll, pitch, and yaw. High-frequency noise (frequencies greater than $1 / 1000$ $\mathrm{m}^{-1}$ ) is lower than 0.01 pixels; low-frequency noise may exceed $1 \mathrm{~m}$.

measured offsets should be zero (tectonic afterslip in the time span between the acquisition of those two images is negligible ${ }^{5}$ ). The large differences in solar angles for those images yield differences in shadowing that may result in located noise in the offsets. However, the areas with difference in shadowing represent less than $0.15 \%$ of the total scene and then do not have a significant influence on the measurements. The spectrum of the measured offset field (computed from the FFT) shows that the lowfrequency bias reaches 0.2 pixels, whereas the highfrequency bias is $\sim 0.01$ pixels (Fig. 7).

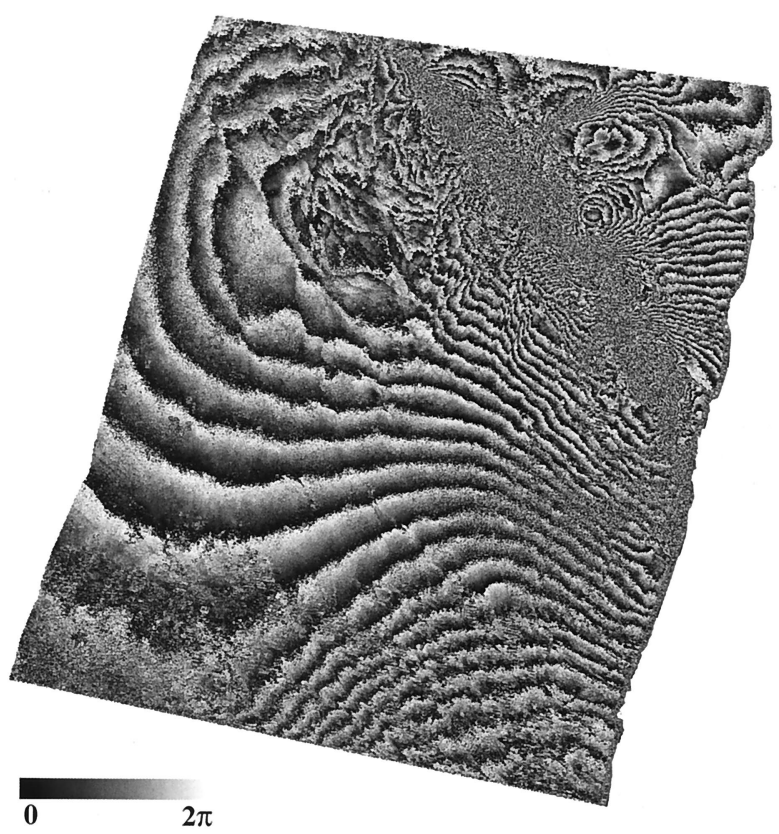

Fig. 8. SAR interferogram showing the near-vertical component of the ground displacement induced by the Landers earthquake. ${ }^{12}$ A fringe represents a near-vertical displacement of $2.8 \mathrm{~cm}$. Nearfault measurement is not available because of signal saturation and decorrelation. 

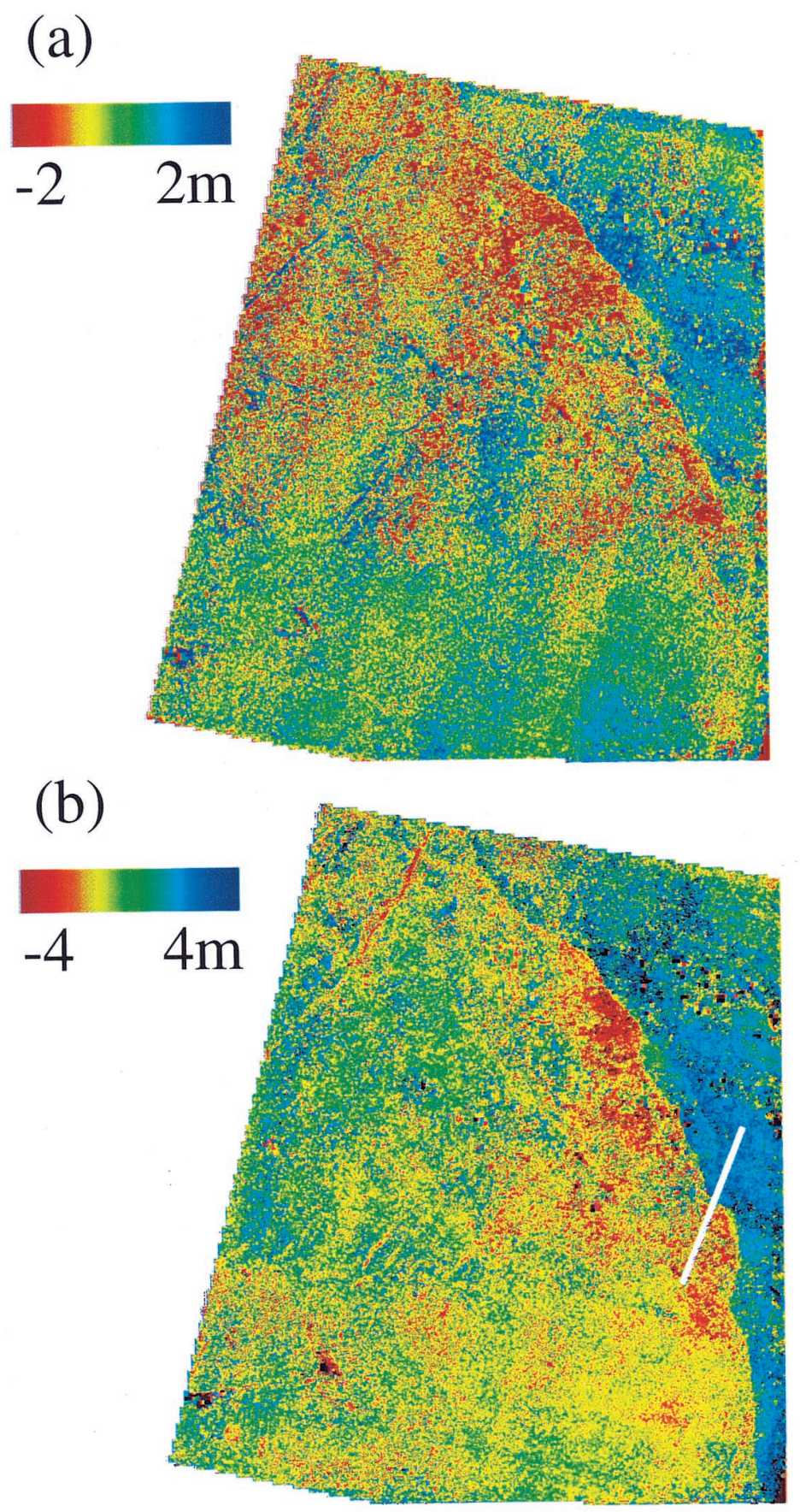

Fig. 9. Ground displacement induced by the Landers earthquake measured from images 1 and 2 (Table 1). (a) East-West and (b) North-South components. Cartography of rupture fits ground measurements to within $\sim 80 \mathrm{~m}$ (Fig. 4). Near-fault measurements allow for direct estimate of amplitude of faulting. Far-field, low-frequency measurements include noise with an amplitude of $\sim 1 \mathrm{~m}$.

\section{Application to the Landers Earthquake}

The Landers earthquake (7.3 Mw, June 1992, California) produced metric surface displacements in an arid area. The fault is $\sim 80 \mathrm{~km}$ long, orientated North-South, and produced right lateral displacement (strike slip fault). It has been extensively studied, and various data are available, including geodetic measurements, ${ }^{6}$ meter scale cartography of surface ruptures, ${ }^{5}$ and SAR interferograms. ${ }^{2}$ SAR interferometry allows for far-field measurements at the centimeter scale but does not provide measurements near the fault (Fig. 8). ${ }^{12}$ We used the images noted in Table 1 to measure the ground displacement according to the technique described above to get the results shown in Fig. 9. The correlation window is $16 \times 16$ pixels wide. Figures $9(\mathrm{a})$ and $9(\mathrm{~b})$ are the East-West and the North-South components, respectively, of the displacement fields. They show a discontinuity that locally follows the map of surface fault ruptures ${ }^{5}$ with an accuracy of $\sim 80 \mathrm{~m}$ [Fig. 4(a)]. 

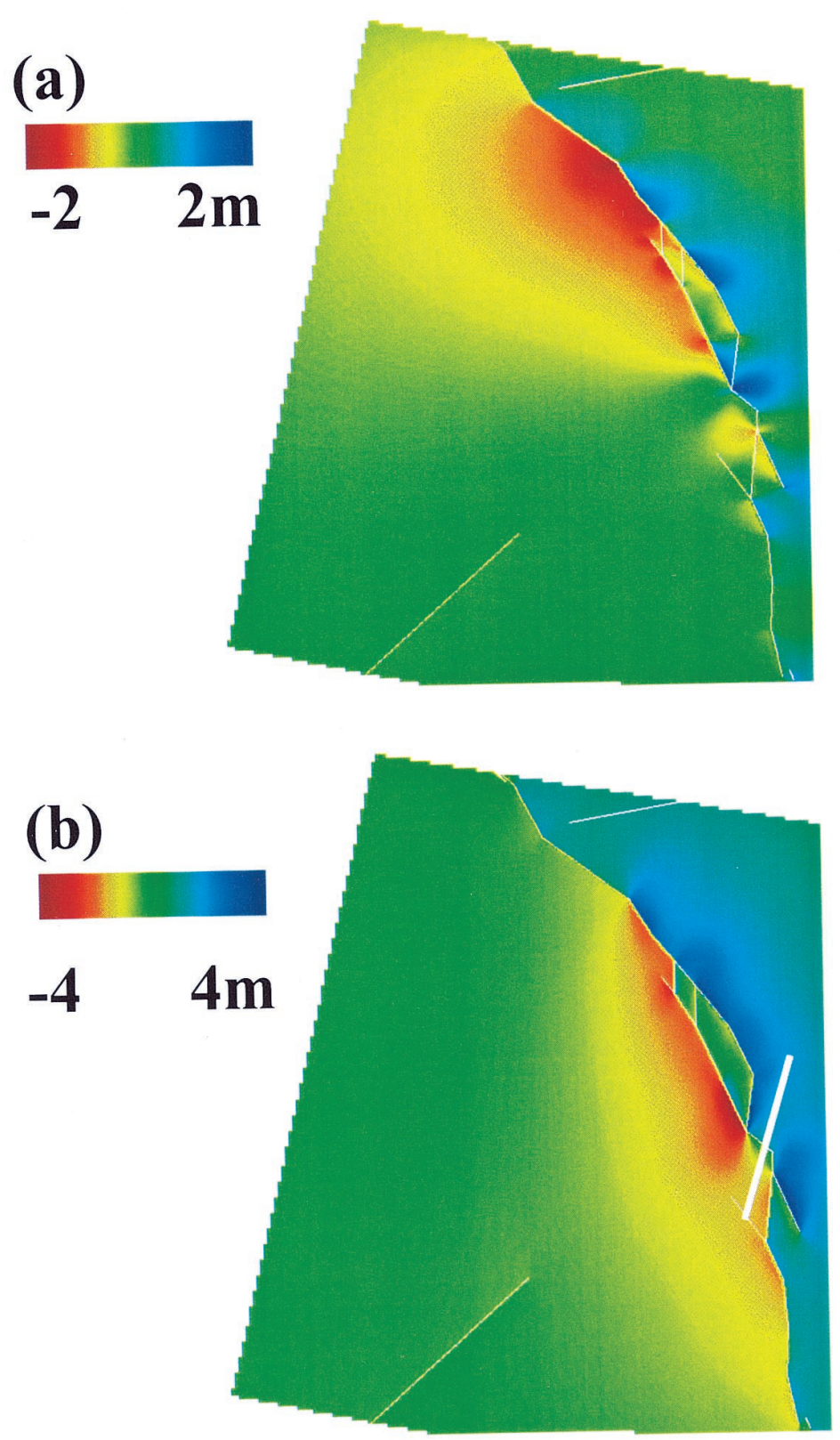

Fig. 10. East-West and North-South elastic modeling of ground displacement constrained by numerous geodetic measurements. ${ }^{6}$

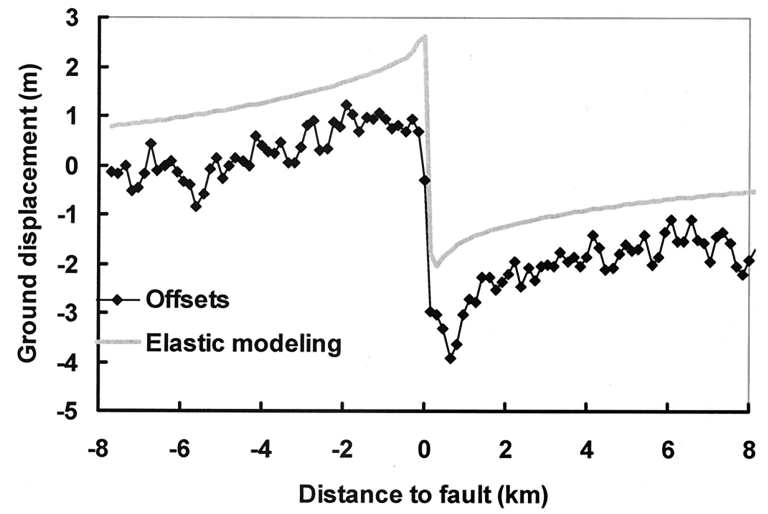

Fig. 11. Profile in offsets and elastic model of Figs. 9 and 10 show good agreement. Ground displacement on the fault can be measured on offsets with an accuracy of $\sim 20 \mathrm{~cm}$.
Measurements are fairly consistent with an elastic modeling of the ground deformation that was constrained from numerous geodetic data (Figs. 10 and 11). 6

Profiles in the offset field (Fig. 11) yield the measurement of the two-dimensional displacement on the fault with an accuracy of $\sim 20 \mathrm{~cm}$. We can reduce that value to few centimeters by averaging profiles across the fault, assuming that the ground displacement does not vary significantly along the average segment.

\section{Conclusions}

Ground displacements induced by an earthquake can be measured from SPOT panchromatic imagery with compensation of other sources of image deformation 
and a dedicated subpixel correlator. When we use a DEM with an accuracy better than $20 \mathrm{~m}$ (rms), the technique provides near-fault measurement with an accuracy of $\sim 10 \mathrm{~cm}$ and low-frequency measurement with an accuracy of $\sim 1 \mathrm{~m}$. The technique is limited mainly by the decorrelation of the images; the accuracy of the DEM; the aliasing of the images; and the uncertainties on the measured roll, pitch, and yaw of the satellite. This technique could also be used to compute DEM from images with a small base-toheight ratio, compensating the low stereoscopic effect by accurate subpixel measurements of offsets. This new application of satellite optical images may gain further interest with the introduction of meter-scale satellite imagery including investigation of earthquakes with low magnitude.

\section{References}

1. D. Massonnet, M. Rossi, C. Carmona, F. Adragna, G. Peltzer, K. Feigl, and T. Rabaute, "The displacement field of the Landers earthquake mapped by radar interferometry," Nature (London) 364, 138-142 (1993).

2. R. Michel, J. P. Avouac, and J. Taboury, "Measuring ground displacements from SAR amplitude images: application to the Landers earthquake," Geophys. Res. Lett. 26, 875-878 (1999).

3. R. Michel and E. Rignot, "Flow of Glaciar Moreno, Argentina, from repeat-pass Shuttle Imaging Radar images: comparison of the phase correlation method with radar interferometry," J. Glaciol. 45, 93-99 (1999).

4. R. E. Crippen, "Measuring of subresolution terrain displace- ments using SPOT panchromatic imagery," Episodes 15, 56-61 (1992).

5. K. Sieh, L. Jones, E. Hauksson, K. Hudnut, D. EberhartPhillips, T. Heaton, S. Hough, K. Hutton, H. Kanamori, A. Lilje, S. Lindvall, S. McGill, J. Mori, C. Rubin, J. Spotila, J. Stock, H. K. Thio, J. Treiman, B. Wernicke, and J. Zachariasen, "Near field investigations of the Landers earthquake sequence, April to July 1992," Science 260, 171-176 (1993).

6. K. W. Hudnut, Y. Bock, M. Cline, P. Fang, Y. Feng, J. Freymueller, X. Ge, W. K. Gross, D. Jackson, M. Kim, N. E. King, J. Langbein, S. C. Larsen, M. Lisowski, Z. K. Shen, J. Svarc, and J. Zhang, "Coseismic displacements of the 1992 Landers earthquake sequence,” Bull. Seismol. Soc. Am. 84, 625-645 (1994).

7. CNES, "Guide des utilisateurs de données SPOT," Vol. 1, "Manuel de référence"; Vol. 2, "Guide de l'utilisateur"; Vol. 3, "Guide pratique" (Centre National d'Etudes Spatiales, Toulouse, France, 1996).

8. J. P. Muller and T. Day, "Digital elevation model production by stereo-matching spot image-pairs: a comparison of algorithms," Image Vision Comput. 7, 2 (1994).

9. L. G. Brown, "A survey of image registration techniques," ACM Comput. Surv. 24, 325-376 (1992).

10. R. Kotynski and K. Chalinska-Macukov, "Optical correlator with dual non-linearity,” J. Mod. Opt. 43, 295-310 (1996).

11. T. Y. Chen, A. C. Bovik, and L. K. Cormack, "Stereoscopic ranging by matching image modulations," IEEE Trans. Image Process. 8, 785-797 (1999).

12. R. Michel, J. P. Avouac, and J. Taboury, "Measuring near field ground displacements from SAR images: application to the Landers earthquake," Geophys. Res. Lett. 26, 3017-3020 (1999). 